\title{
Predictors of Ovarian Reserve and Ovarian Stimulation Outcome in Infertile Women
}

\author{
Juliano Brum Scheffer* \\ Brazilian Institute of Assisted Reproduction, Belo Horizonte, Brazil \\ *Corresponding author: Juliano Brum Scheffer, Scientific Director of Ibrra, Brazilian Institute of Assisted Reproduction, Belo Horizonte, Brazil. \\ Submission: 漈 December 04, 2017; Published: 眥 December 08, 2017
}

\begin{abstract}
Opinion
The fecundity of women begins to decrease after the age of 30 years, primarily as a result of a decrease in the proportion of normal eggs available, which in turn is a consequence of a continuous process of oocyte atresia. Although all women experience this decrease in fecundity, it is difficult to predict the pace of reproductive decline in an individual woman. This age-related decline in fecundity is characterized by a decrease in both egg quality and number, and a population-based change in the expression of markers of ovarian activity, such as a gradual increase in circulating FSH and decreases in circulating anti-Müllerian hormone (AMH) and inhibin B concentrations.
\end{abstract}

A classic report on the effect of female age on fertility found that the percentage of women, who using no contraception remained childless, were increased steadily according to their age of marriage: $6 \%$ at the age of $20-24$ years, $9 \%$ at the age of $25-29$ years, $15 \%$ at the age of $30-34$ years, $30 \%$ at the age of $35-39$ years and $64 \%$ at the age of 40-44 years. The age-associated decline in female fecundity as well as increased risk for spontaneous abortion, are largely attributable to abnormalities in the oocyte. The meiotic spindle in the oocytes of older women frequently exhibits abnormalities in chromosome alignment and microtubular matrix composition. Higher rates of single chromatid abnormalities in oocytes, as well as aneuploidy in preimplantation embryos and ongoing pregnancies, are observed in older women. The higher rate of aneuploidy is a major cause of increased spontaneous abortion and decreased live birth rates in women of advanced reproductive age.

Evaluation of ovarian reserve has been the focus of substantial amount of clinical research during the past several years. AntiMüllerian hormone (AMH), also called Müllerian Inhibiting substance, is a dimeric glycoprotein, belonging to the transforming growth factor-b (TGF-b) super family, such as activins and inhibins, and is produced exclusively in the gonads, as shown more than two decades ago in animals and later in humans. In women, AMH is synthetized by the granulosa cells (GC) surrounding preantral and small antral follicles. Despite the use of ultrasensitive assays,
$\mathrm{AMH}$ is barely detectable in the serum at birth. It reaches higher levels after puberty and then declines with advancing female age, until becoming undetectable again at the time of the menopause. Although its physiological roles and the mechanisms involved in the regulation of $\mathrm{AMH}$ still remain ill-established, recent studies have pointed out this hormone as an attractive marker for assessing of ovarian activity.

Basal AMH, determined before stimulation (usually cycle day 3), was found to be a better measure for the assessment of a decreased ovarian reserve when compared to the classic parameters such as an increase in follicle stimulating hormone (FSH), the decrease of inhibin B, or the antral follicle count. It has also been shown that $\mathrm{AMH}$ was inversely correlated, in addition to age, to basal FSH values. In assisted reproductive technology (ART), serum AMH has also been shown to be the more reliable hormonal marker of the ovarian response to controlled ovarian hyperstimulation $(\mathrm{COH})$ with gonadotropins than the basal levels of FSH, estradiol, inhibin $\mathrm{B}$, or the woman's age. Also, AMH has been claimed to possess at least the same level of accuracy as the antral follicle count (AFC) for the prediction of poor and excessive response. In addition, a high serum concentration of AMH before the start of $\mathrm{COH}$ has been shown to be associated with increased risk of developing ovarian hyperstimulation syndrome (OHSS).

The ideal ovarian reserve test should be reproducible, with limited inter- and intra-cycle variability, and demonstrate high specificity to minimize the risk for incorrectly categorizing women as having decreased ovarian reserve. No measure of ovarian reserve is perfect; however, AMH level and age have good predictive value. More studies have to be done to improve the accuracy and interpretation of the current ovarian reserve markers to state clear cut-off levels for each markers and find another markers which could more correlate with the number of ova retrieved, embryo quality and clinical pregnancy rate. Determining the etiology of maternal aging on oocyte competence could lead to improve patient care and fertility outcome. 